\title{
Focal beam position detection in a laser Induced Breakdown Spectroscopy system by using a Fiber Bragg Grating sensor
}

\author{
F. Anabitarte* a, L. Rodriguez-Cobo ${ }^{\text {a }}$, C. Galindez ${ }^{\text {a }}$, A. Ullan ${ }^{\text {a }}$, A. Cobo ${ }^{\text {a }}$ \\ ${ }^{a}$ Photonic Engineering Group, University of Cantabria, Avda. Los Castros s/n, 39005 \\ Santander, Spain.
}

\begin{abstract}
Chemical analysis of dangerous materials entails a safety issue for the researchers. Laser Induced Breakdown Spectroscopy offers the possibility to analyze these materials away from them using StandOff Set-ups. To optimize the plasma induction, the remote focalization of the laser beam is of paramount importance. A custom Fiber Bragg Grating sensor system able to correct the laser beam focalization errors is proposed and experimentally checked. The optical transducer architecture and the preliminary obtained results are reported in this paper.
\end{abstract}

Keywords: Laser Induced Breakdown Spectroscopy, Fiber Bragg Grating, focalization

\section{INTRODUCTION}

Laser Induced breakdown Spectroscopy (LIBS) is a class of atomic emission spectroscopy that provides a fast analysis of chemical composition of a big range of solid, liquid or gas samples. LIBS uses a high energy laser pulse to excite and vaporize the sample, inducing plasma. Each atomic specie emits different wavelengths and the light emitted from the plasma is acquired with an optical system and diffracted by a spectrometer, generating a "spectral fingerprint" of the sample [1,2].

LIBS has increased its applications fields quickly [2,3]. Among these fields, applications as different as archeology [4], art [5], heavy metals [6] or explosive trace detection are in this big group. Some applications implies to work with dangerous samples or in dangerous environments. Stand-off LIBS setups [7], where the LIBS devices are far from the sample, emerged under the coverage of these kind of experiments with dangerous materials.

To induce optimum spectroscopic plasma behaviors using Stand-off LIBS set-ups, the laser pulse focalization on the sample is, today, an open topic. Plasma features are strongly dependent of many factors like matrix effects [8], laser pulse energy or lens to sample distance (LTSD) [9]. All these factors and others are related to each other, inducing different plasma features for each set of them. A wrong focalization on the sample surface can change the irradiance on the sample, affecting to the "spectral fingerprint" [9]. There is an optimal position to LTSD, but if the distance between the sample and the LIBS set-up is in the order of several meters, like in Stand-off configurations, it can be difficult to find it. The measurement of the acoustic wave generated by the plasma has been proposed as a monitoring signal of the ablation process, as its intensity or spectral content can be related to the plasma emission. It is found that after the laser pulse-sample interaction, the intensity of the induced shockwave varies with the focal position, which can be used to obtain an error signal to reach optimums LTSD's.

In this work, a new prototype based on a Fiber Bragg Grating (FBG) to detect and quantify the shockwave induced by the plasma expansion [10-12] is proposed. This FBG sensor is built in an optical fiber which allows the user to place the sensor close to the sample and far away from the instrumentation, avoiding the hazards due to the sample or the environment. The transducer architecture and the experimental preliminary results are reported and discussed in this paper.

* anabitartef@unican.es; phone 34 942200877; fax 34942200877 


\subsection{LIBS set-up}

\section{EXPERIMENTAL SET-UP}

The LIBS set-up used on these experiments is shown on Figure 1.

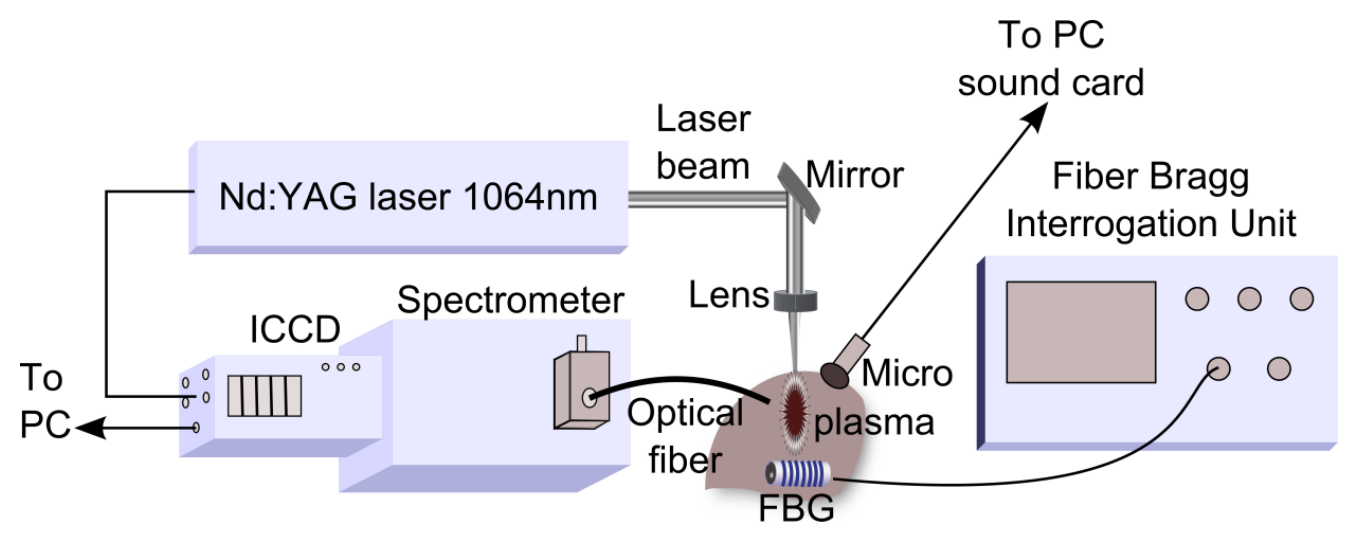

Figure 1: Experimental set-up

A pulsed LS-2147 (Lotis TII) laser source, with a wavelength of $1064 \mathrm{~nm}$, is used to induce the plasma. The laser provides optical pulses of $100 \mathrm{~mJ}, 16 \mathrm{nseg}$ time at a frequency rate of $10 \mathrm{~Hz}$. A lens of $75 \mathrm{~mm}$ of focal length is used to focalize the laser on the sample surface. The sample is placed on a linear stage to provoke the focalization or the de-focalization of the laser beam on its surface. An optical fiber with a core diameter of $600 \mu \mathrm{m}$ is positioned in front of the plasma to capture and drive the light into the spectrometer. The spectrometer is a SpectraPro 250i (Princeton Instruments), with a grating of 12001/mm blazed at $450 \mathrm{~nm}$ and centered at $450 \mathrm{~nm}$. This device diffracts the light in the range between $304 \mathrm{~nm}$ and $581 \mathrm{~nm}$ wavelengths and it is acquired with an intensified charge-coupled device (iCCD) (PIMAX 3, Princeton Instruments) that works as an optical spectrum analyzer. The iCCD is equipped with a time control unit which triggers the laser and acquires the spectrum $4 \mu$ seg after the laser shot during $5 \mu$ seg in order to avoid the continuum radiation. The obtained spectra are processed with a computer and WinSpec (Princeton Instruments) software.

It is well known that the first life stages of plasma are dominated by a continuum emission which can hide the atomic emission lines [2] and that the provoked acoustic emissions emission intensity can also be used to estimate the optimization of the laser-sample interaction [11]. The shockwave induces a pressure wave whose energy depends on plasma emission intensity. To check the proposed FBG behaviors, a calibrated microphone (Moving coil dynamic element, Hyper-Cardioid MICPRO7N HQ Power) has been used. Both the microphone and the FBG have been positioned close to the crater induced by the laser $(200 \mathrm{~mm}$ and $50 \mathrm{~mm}$ respectively).

\subsection{Fiber Bragg Grating sensor}

The FBG transducer scheme is shown in Figure 2. A FBG $\left(\lambda_{\text {Bragg }}=1541 \mathrm{~nm}\right)$ has been fixed to an acetate sheet $10 \mathrm{~mm}$ wide $20 \mathrm{~mm}$ long in order to improve the mechanical response with a bigger contact surface and without adding a big weight. The optical fiber has been glued to a PVC frame (27 $\mathrm{mm}$ width $140 \mathrm{~mm}$ length) with the fixing points as close as possible to the end of acetate sheet (28mm between them) to improve its frequency response.

The signal from the FBG was monitorized with a FBG interrogation unit (Braggscope, Fiber Sensing) which is able to work properly up to $10000 \mathrm{~Hz}$. The acquisition rate is important is this case because the plasma and the shockwave lifetimes are short, in the order of several microseconds, and in consequence slower acquisition rates can induce a subsampling effect and distort the shockwave signal.

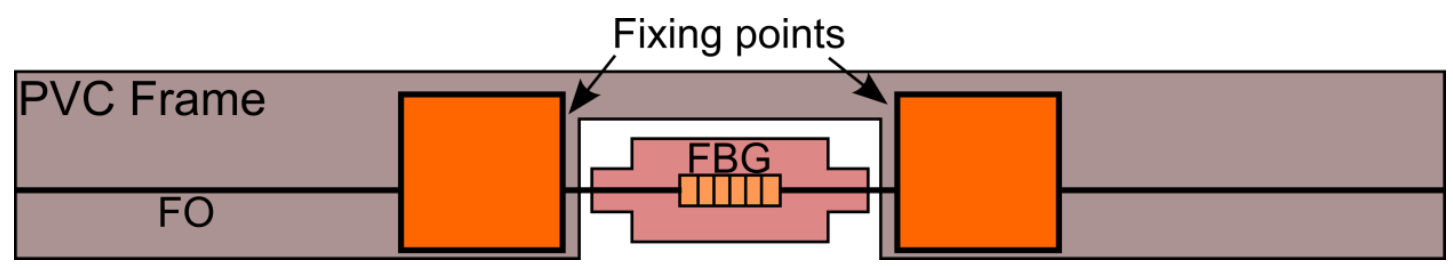

Figure 2: FBG sensor detail 


\section{RESULTS AND ITS DISCUSSION}

The sample, a leaded brass plate, was in the center of focal position range of the laser focalization lens. It is important to note that the focal position is not a concrete distance. Laser beam focalization process induces plasma in a range of distance around the lens focal length because plasma threshold is overcome into the surrounding distance around focal length and the lens that focuses the laser beam has aberrations. The linear stage moves the sample closer and further the lens focal plane center in a range between $10 \mathrm{~mm}$ up and $7.5 \mathrm{~mm}$ down of this optimal focal plane in steps of $2.5 \mathrm{~mm}$. FBG and microphone are fixed to this linear stage in order to maintain the same position for all points. 220 spectra were acquired for each position. Each spectrum has associated a shockwave measurement acquired with both the FBG sensor and the microphone. The first 20 spectra are rejected in order to remove impurities on sample surface. After obtaining these values, the results of the spectra are averaged for each position in order to analyze the variation of the shockwave measurements. Obtained shockwave measurements from the calibrated microphone and from the proposed FBS sensors are both summarized in Figure 3. The admitted deviations around the expected optimum focal range are also shown in the figure with two vertical dotted lines.

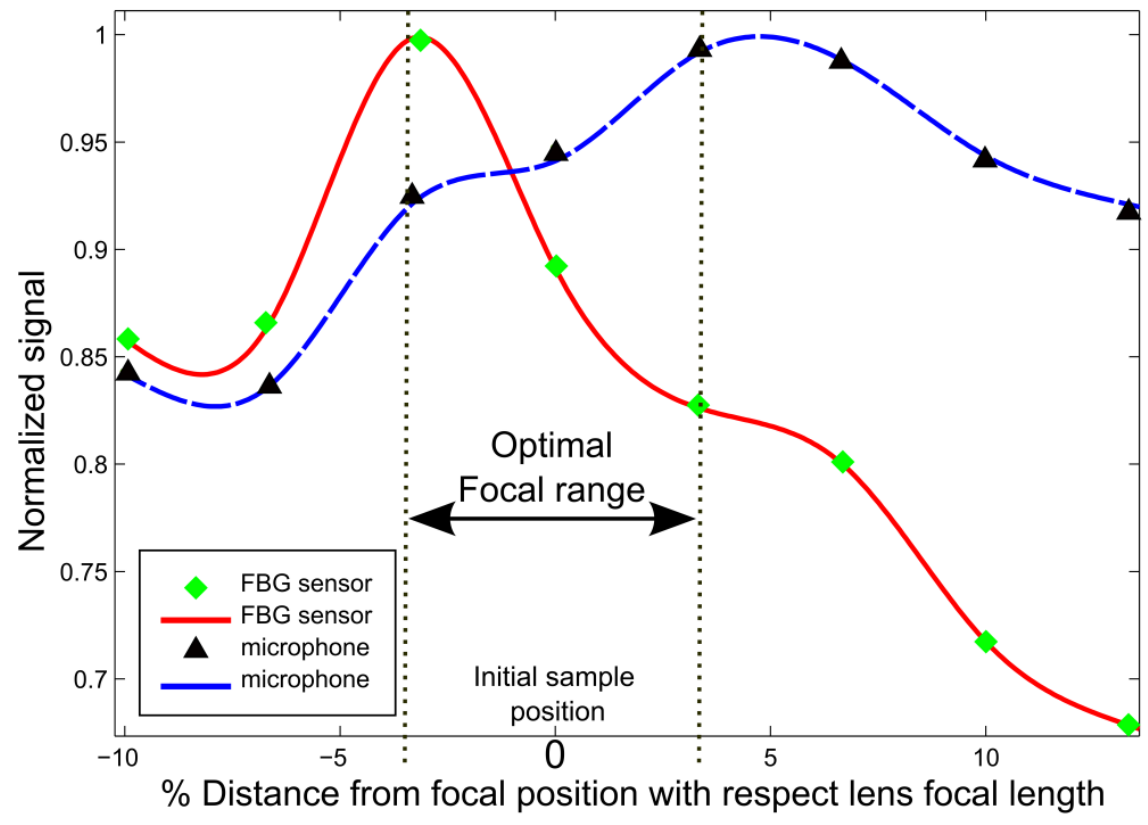

Figure 3: Results of FBG sensor (solid red line) and microphone (dotted blue line)

Both microphone and FBG exhibit different behaviors. FBG shows a maximum centered about $2.5 \mathrm{~mm}$ below the lens focal plane and the microphone peak is around $3 \mathrm{~mm}$ above the lens focal plane. At the same time, FBG maximum is clearer and acuter than microphone peak and it is into the optimal focal range. In order to correct the focal position, FBG shows better accuracy and despite the facts that the maximum is not the initial focal position, the error is within the limits. The LTSD changes the irradiance (laser power per unit of sample surface) and these changes in the irradiance change the plasma shape [10]. The plasma size and shape are related to the compression of the surrounding medium and this compression changes the induced shockwave. The biggest the irradiance, the biggest the plasma plume and the shockwave. When LTSD is reduced, the sample surface irradiated for the laser is bigger and consequently the irradiance is lower. This effect reduces the induced shockwave. Contrarily, if LTSD is increased the plasma is induced above the sample surface. This reduces the ablated material but the laser is correctly focalized above the sample. Then, the laser ionizes the air and in theory the plasma is induced in the focal position with the maximal irradiance. This effect should induce the best possible shockwave but at the same time the breakdown threshold (the irradiance that a material needs to be ionized) of the air is bigger, generating a small plasma plume and shockwave.

The features of FBG sensor and FBG interrogation unit allow a sample rate of 10000 samples per second. The microphone allows a sample rate of 44100 samples per second. This interrogation unit feature reduces the spectral response of the FBG sensor with respect the microphone, inducing a detection 
dominated by mechanical effects and with aliasing troubles. This features changes the maximal response point of each sensor although the best point for each sensor is close or inside the focal optimal range. It should be taken into account that these displacements are small with respect the lens focal distance $(75 \mathrm{~mm})$ and both sensors allow the focal point detection with an error around $3 \%$.

\section{CONCLUSIONS}

To detect and quantify the shockwave induced by the plasma expansion, a new prototype based on a Fiber Bragg Grating (FBG) is proposed in this paper. This FBG sensor is able to be placed close to the sample and far away from the instrumentation, avoiding the hazards due to the sample or the environment.

These preliminary experimental results suggest the feasibility of this sensor proposal for the correction of laser beam focalization errors. Ongoing works are in course to obtain a more optimized sensor configuration to reach lower focalization errors which will enable high precision and remote controlled stand-off LIBS set-ups working into dangerous samples or environments.

\section{ACKNOWLEDGMENTS}

This work has been co-supported by the Spanish Government through the project TEC2010-20224-C0202 and the grant AP2007-02230.

\section{REFERENCES}

[1] A. W. Miziolek, V. Palleschi, and I. Schechter, [Laser-induced breakdown spectroscopy (LIBS): fundamentals and applications] Cambridge Univ Pr, (2006).

[2] B. Kearton, and Y. Mattley, "Laser-induced breakdown spectroscopy: Sparking new applications," Nature Photonics, 2(9), 537-540 (2008).

[3] R. W. Bogue, "Boom time for LIBS technology," Sensor Review, 24(4), 353-357 (2004).

[4] L. Torrisi, F. Caridi, L. Giuffrida et al., "LAMQS analysis applied to ancient Egyptian bronze coins," Nuclear Instruments and Methods in Physics Research Section B: Beam Interactions with Materials and Atoms, 268(10), 1657-1664 (2010).

[5] M. Ferretti, G. Cristoforetti, S. Legnaioli et al., "In situ study of the Porticello Bronzes by portable X-ray fluorescence and laser-induced breakdown spectroscopy," Spectrochimica Acta Part B: Atomic Spectroscopy, 62(12), 1512-1518 (2007).

[6] N. K. Rai, and A. Rai, "LIBS--an efficient approach for the determination of Cr in industrial wastewater," Journal of hazardous materials, 150(3), 835-838 (2008).

[7] C. Lopez-Moreno, S. Palanco, J. J. Laserna et al., "Test of a stand-off laser-induced breakdown spectroscopy sensor for the detection of explosive residues on solid surfaces," J. Anal. At. Spectrom., 21(1), 55-60 (2005).

[8] A. S. Eppler, D. A. Cremers, D. D. Hickmott et al., "Matrix effects in the detection of Pb and Ba in soils using laser-induced breakdown spectroscopy," Applied Spectroscopy, 50(9), 1175-1181 (1996).

[9] J. Aguilera, C. Aragon, and F. Penalba, "Plasma shielding effect in laser ablation of metallic samples and its influence on LIBS analysis," Applied surface science, 127, 309-314 (1998).

[10] S. Conesa, S. Palanco, and J. Laserna, "Acoustic and optical emission during laser-induced plasma formation," Spectrochimica Acta Part B: Atomic Spectroscopy, 59(9), 1395-1401 (2004).

[11] A. Hrdlicka, L. Zaorálková, M. Galiová et al., "Correlation of acoustic and optical emission signals produced at 1064 and $532 \mathrm{~nm}$ laser-induced breakdown spectroscopy (LIBS) of glazed wall tiles," Spectrochimica Acta Part B: Atomic Spectroscopy, 64(1), 74-78 (2009).

[12] J. M. Lopez-Higuera, [Handbook of optical fibre sensing technology] John Wiley \& Sons Inc, (2002). 